\title{
Consecutive patterns in permutations: clusters and generating functions
}

\author{
Sergi Elizalde ${ }^{1}$ and Marc Noy ${ }^{2}$ \\ ${ }^{1}$ Department of Mathematics, Dartmouth College, Hanover, NH 03755. \\ ${ }^{2}$ Departament de Matemàtica Aplicada II, Universitat Politècnica de Catalunya, Barcelona, Spain.
}

\begin{abstract}
We use the cluster method in order to enumerate permutations avoiding consecutive patterns. We reprove and generalize in a unified way several known results and obtain new ones, including some patterns of length 4 and 5 , as well as some infinite families of patterns of a given shape. Our main tool is the cluster method of Goulden and Jackson. We also prove some that, for a large class of patterns, the inverse of the exponential generating function counting occurrences is an entire function, but we conjecture that it is not D-finite in general.

Résumé. On utilise la méthode des clusters pour énumérer permutations qui évitent motifs consécutifs. On redémontre et on généralise d'une manière unifiée plusieurs résultats et on obtient de nouveaux résultats pour certains motifs de longueur 4 et 5 , ainsi que pour certaines familles infinies de motifs. L'outil principal c'est la méthode des clusters de Goulden et Jackson. On démontre aussi que, pour une grande classe de motifs, l'inverse de la série génératrice exponentielle qui compte occurrences est une fonction entière, mais on conjecture qu'elle n'est pas D-finie en général.
\end{abstract}

Keywords: Pattern avoidance; Consecutive patterns; Cluster method.

\section{Introduction}

Consecutive patterns in permutations are a natural variation of the classical definition of patterns. Consecutive patterns arise naturally when studying descents, double descents, peaks, and alternating permutations. The systematic study of consecutive patterns in permutations was started by the authors in [6], and several papers have been written on the topic since then. In this paper we use the cluster method of Goulden and Jackson in order to obtain new results on avoiding consecutive patterns in permutations. Given a pattern $\sigma$, the method consists of counting partial permutations in which each element is involved in at least one occurrence of $\sigma$, the so-called clusters. By inclusion-exclusion, the enumeration of clusters provides the enumeration of permutations according to the number of occurrences of $\sigma$.

Counting clusters can be seen as counting linear extensions in a certain poset. For instance, if $\sigma$ is the monotone pattern, the corresponding poset is simply a chain, and counting linear extensions is a trivial task. It follows that not only the monotone pattern $12 \cdots s$ can be analyzed in this way, but also the pattern $123 \cdots(s-1)(s+1) s(s+2)(s+3) \cdots 2 s$ and other related ones, which we call chain patterns. Another significant case is when $\sigma$ is a non-overlapping pattern, which means that two occurrences of $\sigma$ in a permutation cannot overlap in more than one position. By analyzing the associated poset when $\sigma_{1}=1$, we prove that if $\sigma=1 \sigma_{2} \cdots \sigma_{m}$ is non-overlapping, then the number of permutations avoiding $\sigma$ depends 
only on the value $b=\sigma_{m}$, and the inverse $w(z)$ of the associated exponential generating function satisfies a linear differential equation of order $b$. This generalizes a result proved in [6] using a representation of permutations as increasing binary trees. For chain patterns and non-overlapping patterns, the differential equations that we obtain can also be deduced from the work of Khoroshkin and Shapiro [8].

A more intricate example is the pattern 1324. This case was left open in [6], and it cannot be solved with the techniques from [8] either. The number of linear extensions of the associated poset is a Catalan number and this allows us to prove in this case that the inverse of the generating function satisfies a linear differential equation of order five with polynomial coefficients. Again, the technique can be extended to cover the general pattern $134 \cdots(s+1) 2(s+2)(s+3) \cdots 2 s$. For other patterns, such as 1423 and 2143 , we find recurrence relations satisfied by the cluster numbers, but we are not able to find a closed solution in terms of differential equations. In fact, we conjecture that the inverse of the generating function for permutations avoiding 1423 is not D-finite. If true, this conjecture would give the first instance of a pattern with this property, and it would make a related conjecture of Noonan and Zeilberger for classical patterns less believable.

The present situation for small patterns is the following. There are two inequivalent patterns of length 3 , already solved in [6]. There are 7 inequivalent patterns of length 4 , and we still do not have closed solutions for 1423, 2143 and 2413. There are 25 inequivalent patterns of length 5, among which we can solve only a few. For patterns of length 6, we prove four conjectures of Nakamura [11] regarding the equivalence of certain pairs, completing the classification and proving that there are exactly 92 inequivalent patterns. Similar techniques can be used to study permutations avoiding more than one pattern. See [8] for work in this direction.

We finish this section with some definitions and preliminaries. In Sections 2 and 3 we study monotone and non-overlapping patterns. Section 4 is devoted to the pattern 1324 and generalizations, and Section 5 to other patterns of length four. We conclude with some results on asymptotics, specially the fact that the monotone pattern dominates all non-overlapping patterns, confirming in this case a conjecture of Elizalde and Noy [6]. Due to lack of space, several results are presented without proofs.

\subsection{Consecutive patterns}

Given a sequence of distinct positive integers $\tau=\tau_{1} \cdots \tau_{k}$, we define the reduction $\operatorname{st}(\tau)$ as the permutation of length $k$ obtained by relabelling the elements of $\tau$ with $\{1, \ldots, k\}$ so that the order relations among the elements remains the same. For instance st $(46382)=34251$. Given permutations $\pi \in \mathcal{S}_{n}$ and $\sigma \in \mathcal{S}_{m}$, we say that $\pi$ contains $\sigma$ as a consecutive pattern if $\operatorname{st}\left(\pi_{i} \cdots \pi_{i+m-1}\right)=\sigma$ for some $i \in\{1, \ldots, n-m+1\}$. We denote by $c_{\sigma}(\pi)$ the number of occurrences of $\sigma$ in $\pi$ as a consecutive pattern, and by $\alpha_{n}(\sigma)$ be the number of permutations in $\mathcal{S}_{n}$ that avoid $\sigma$ as a consecutive pattern. In this paper, containment and avoidance always refer to consecutive patterns.

Let

$$
P_{\sigma}(u, z)=\sum_{n \geq 0} \sum_{\pi \in \mathcal{S}_{n}} u^{c_{\sigma}(\pi)} \frac{z^{n}}{n !}
$$

be the bivariate exponential generating function for occurrences of $\sigma$ in permutations. It will be convenient to define $\omega_{\sigma}(u, z)=1 / P_{\sigma}(u, z)$. Note that

$$
P_{\sigma}(0, z)=\frac{1}{\omega_{\sigma}(0, z)}=\sum_{n \geq 0} \alpha_{n}(\sigma) \frac{z^{n}}{n !}
$$


is the generating function of permutations avoiding $\sigma$. We will remove the subscript $\sigma$ from $P$ and $\omega$ when the pattern is clear from the context. If $\Sigma$ is a set of patterns, we define $P_{\Sigma}(u, z)$ and $\omega_{\Sigma}(u, z)$ similarly, where $u$ marks the total number of occurrences of all the patterns in $\Sigma$.

Two occurrences of $\sigma$ in a permutation may overlap in certain positions. This motivates the following definition. Let $O_{\sigma}$ be the set of indices $i$ with $1 \leq i<m$ such that $\operatorname{st}\left(\sigma_{i+1} \sigma_{i+2} \ldots \sigma_{m}\right)=$ $\operatorname{st}\left(\sigma_{1} \sigma_{2} \ldots \sigma_{m-i}\right)$. Equivalently, $i \in O_{\sigma}$ if there is some permutation in $\mathcal{S}_{m+i}$ where both its leftmost $m$ entries and its rightmost $m$ entries form occurrences of $\sigma$ (these occurrences overlap in exactly $m-i$ positions). We call $O_{\sigma}$ the set of overlaps of $\sigma$. Note that if $m \geq 2$, then $m-1 \in O_{\sigma}$.

Given a pattern $\sigma_{1} \cdots \sigma_{m}$, its reversal is $\sigma_{m} \cdots \sigma_{1}$, and its complementation is $\left(m+1-\sigma_{1}\right) \cdots(m+$ $\left.1-\sigma_{m}\right)$. Reversal and complementation do not change the distribution of occurrences of a pattern. Patterns of small length were first studied in [6]. It was shown that patterns of length three fall into two classes, represented by 123 and 132, and the associated generating functions $P_{\sigma}(u, z)$ were computed explicitly. Patterns of length four fall into seven classes, and three of them were solved in [6] in terms of the generating functions, namely 1234, 1342 and 1243 .

\subsection{The cluster method}

The cluster method of Goulden and Jackson [7] is a powerful method for enumerating words with respect to occurrences of certain substrings, based on inclusion-exclusion. Several extensions and implementations of the method have been given in the literature, most notably in [13]. It is also related to the lace expansion of Brydges and Spencer [2]. Let us now summarize an adaptation of the cluster method to the enumeration of permutations with respect to the number of occurrences of a consecutive pattern. This adaptation has been recently used by Dotsenko and Khoroshkin [3], and it has many similarities with a method of Mendes and Remmel [10] based on the combinatorics of symmetric functions.

For fixed $\sigma \in \mathcal{S}_{m}$, a $k$-cluster of length $n$ with respect to $\sigma$ is a pair $\left(\pi,\left(i_{1}, i_{2}, \ldots, i_{k}\right)\right)$ where

- $\pi \in \mathcal{S}_{n}$,

- $1=i_{1}<i_{2}<\cdots<i_{k}=n-m+1$,

- for each $1 \leq j \leq k, \operatorname{st}\left(\pi_{i_{j}} \pi_{i_{j}+1} \ldots \pi_{i_{j}+m-1}\right)=\sigma$,

- for each $1 \leq j \leq k-1, i_{j+1} \leq i_{j}+m-1$.

In other words, the $i_{j}$ are starting positions of occurrences of $\sigma$ in $\pi$, all the entries of $\pi$ are in at least one of the marked occurrences, and neighboring marked occurrences overlap. Note that $i_{j+1}-i_{j} \in O_{\sigma}$ for all $j$, and that $\pi$ may have more than $k$ occurrences of $\sigma$. Sometimes we write $\left(\pi ; i_{1}, i_{2}, \ldots, i_{k}\right)$ instead of $\left(\pi,\left(i_{1}, i_{2}, \ldots, i_{k}\right)\right)$. For example, if $\sigma=1324$, then $(142536879 ; 1,3,6)$ is a 3 -cluster of length 9 , since 1425,2536 and 6879 are occurrences of $\sigma$ and all the entries are in at least one of the occurrences. Notice that the 1425 and 2536 overlap in two positions, whereas 2536 and 6879 overlap only in one position.

Let $r_{n, k}$ be the number of $k$-clusters of length $n$ with respect to a fixed $\sigma$. We denote by

$$
R_{\sigma}(u, z)=\sum_{n, k} r_{n, k} u^{k} \frac{z^{n}}{n !}
$$

the exponential generating function (EGF for short) for clusters, and by

$$
\widehat{R}_{\sigma}(u, x)=\sum_{n, k} r_{n, k} u^{k} x^{n}
$$


the corresponding ordinary generating function (OGF for short). The following theorem, which is an adaptation of [7, Theorem 2.8.6] to the case of permutations, expresses the EGF for occurrences of $\sigma$ as a consecutive pattern in permutations in terms of the EGF for clusters.

Theorem 1.1 ([7]) For any pattern $\sigma$ we have

$$
\omega_{\sigma}(u, z)=1-z-R_{\sigma}(u-1, z) .
$$

In particular, the EGF for $\sigma$-avoiding permutations is

$$
P_{\sigma}(0, z)=\frac{1}{\omega_{\sigma}(0, z)}=\frac{1}{1-z-R_{\sigma}(-1, z)} .
$$

It will be convenient to denote $\widehat{A}(u, x)=1-x-\widehat{R}(u, x)$ and $A(u, z)=1-z-R(u, z)$, so that $\omega_{\sigma}(u, z)=A(u-1, z)$.

Theorem 1.1 reduces the study of the distribution of occurrences of a pattern in permutations to computing the cluster numbers $r_{n, k}$. We now show that these numbers can be expressed in terms of linear extensions of certain posets. Fix $\sigma \in \mathcal{S}_{m}$. For given $n, k$, let

$$
\mathcal{I}_{n, k}^{\sigma}=\left\{\left(i_{1}, i_{2}, \ldots, i_{k}\right): i_{1}=1, i_{k}=n-m+1, \text { and } i_{j+1}-i_{j} \in O_{\sigma} \text { for } 1 \leq j \leq k-1\right\},
$$

and fix $\left(i_{1}, \ldots, i_{k}\right) \in \mathcal{I}_{n, k}^{\sigma}$. A permutation $\pi \in \mathcal{S}_{n}$ has the property that $\left(\pi ; i_{1}, \ldots, i_{k}\right)$ is a $k$-cluster of length $n$ if and only if, for each $1 \leq j \leq k$, we have $\operatorname{st}\left(\pi_{i_{j}} \pi_{i_{j}+1} \ldots \pi_{i_{j}+m-1}\right)=\sigma$. If we denote by $\varsigma \in \mathcal{S}_{m}$ the inverse of $\sigma$, so that $\varsigma_{\ell}$ is the position of $\ell$ in $\sigma$, then this is equivalent to $\pi_{\varsigma_{1}+i_{j}-1}<$ $\pi_{\varsigma_{2}+i_{j}-1}<\cdots<\pi_{\varsigma_{m}+i_{j}-1}$. These conditions for $1 \leq j \leq k$ define a partially ordered set (poset) $P_{n, i_{1}, \ldots, i_{k}}^{\sigma}$ on the entries of $\pi$. For example, the poset $P_{6,1,3}^{1324}$ is defined by $\pi_{1}<\pi_{3}<\pi_{2}<\pi_{4}$ and $\pi_{3}<\pi_{5}<\pi_{4}<\pi_{6}$, and it is drawn in Figure 2 (where $k=2$ ).

If we denote by $\mathcal{L}(P)$ the set of linear extensions (i.e., compatible linear orders) of $P$, then it follows that $\left(\pi ; i_{1}, \ldots, i_{k}\right)$ is a $k$-cluster of length $n$ with respect to $\sigma$ if and only if $\pi \in \mathcal{L}\left(P_{n, i_{1}, \ldots, i_{k}}^{\sigma}\right)$. We denote by $\mathcal{P}_{n, k}^{\sigma}$ the multiset of such posets for all values of $\left(i_{1}, \ldots, i_{k}\right) \in \mathcal{I}_{n, k}^{\sigma}$. Note that some posets in $\mathcal{P}_{n, k}^{\sigma}$ can appear with multiplicity, as in the cases discussed in Section 2 Alternatively, we could mark the elements $\pi_{i_{1}}, \pi_{i_{2}}, \ldots, \pi_{i_{k}}$ in $P_{n, i_{1}, \ldots, i_{k}}^{\sigma}$, to ensure that all the posets in $\mathcal{P}_{n, k}^{\sigma}$ are different as marked posets. We have that

$$
r_{n, k}=\sum_{P \in \mathcal{P}_{n, k}^{\sigma}}|\mathcal{L}(P)| .
$$

We also define the multisets $\mathcal{P}_{n}^{\sigma}=\bigcup_{k \geq 1} \mathcal{P}_{n, k}^{\sigma}$ and $\mathcal{P}^{\sigma}=\bigcup_{n} \mathcal{P}_{n}^{\sigma}$.

\subsection{Ordinary and exponential generating functions}

Here we describe a tool that we use to switch between the OGF and the EGF of a sequence. Let $L$ be the linear operator on formal power series such that $L\left(x^{k}\right)=z^{k} / k$ ! for all $k \geq 0$.

Lemma 1.2 Let $\widehat{A}(x)=\sum_{n \geq 0} a_{n} x^{n}$ be an OGF with corresponding EGF $A(z)=\sum_{n \geq 0} a_{n} z^{n} / n$ !. Let $I$ denote the integral operator with respect to $z$, that is, $I F(z)=\int_{0}^{z} F(v) d v$, and let $j \geq 0$. Then

$$
L\left(x^{j} \widehat{A}\right)=I^{j} A, \quad L\left(x^{j+1} \widehat{A}^{\prime}\right)=I^{j}\left(z A^{\prime}\right), \text { and } \quad L\left(x^{j} \widehat{A}^{(j)}\right)=z^{j} A^{(j)} .
$$

Given a linear differential equation for $\widehat{A}(x)$ with polynomial coefficients, Lemma 1.2 is used to obtain a linear differential equation for $A(z)$ with polynomial coefficients. All the derivatives of multivariate generating functions are always partial derivatives with respect to $z$. Similarly, initial conditions are for $z$. 


\section{Monotone and related patterns}

\subsection{The pattern $\sigma=12 \ldots m$}

For the monotone pattern $\sigma=12 \ldots m$, a differential equation satisfied by $\omega_{\sigma}(u, z)$ was given in [6, Theorem 3.1]. The proof is based on representations of permutations as increasing binary trees.

Theorem $2.1([6])$ Let $m \geq 3$, let $\sigma=12 \ldots m$, and let $\omega(z):=\omega_{\sigma}(u, z)$. Then $\omega$ is the solution of

$$
\omega^{(m-1)}+(1-u)\left(\omega^{(m-2)}+\cdots+\omega^{\prime}+\omega\right)=0
$$

with $\omega(0)=1, \omega^{\prime}(0)=-1, \omega^{(i)}(0)=0$ for $2 \leq i \leq m-2$.

Proof: Let us give an alternative proof using the cluster method. It is clear that $O_{\sigma}=\{1,2, \ldots, m-1\}$, so for $\pi \in \mathcal{S}_{n},\left(\pi ; i_{1}, \ldots, i_{k}\right)$ is a $k$-cluster with respect to $\sigma$ if and only if $\pi_{1}<\pi_{2}<\cdots<\pi_{n}$ and $1 \leq i_{j+1}-i_{j} \leq m-1$ for all $j$. It follows that the OGF for the cluster numbers is

$$
\widehat{R}_{\sigma}(u, x)=\frac{u x^{m}}{1-u\left(x+x^{2}+\cdots+x^{m-1}\right)} .
$$

Clearing denominators in the expression for $\widehat{A}(u, x)=1-x-\widehat{R}_{\sigma}(u, x)$, applying the transformation $L$, and using Lemma 1.2 , we get

$$
\left(1-u\left(I+I^{2}+\cdots+I^{m-1}\right)\right) A(u, z)=1-z-u z .
$$

Differentiating $m-1$ times we obtain a differential equation for $A(z)=A(u, z)$ :

$$
A^{(m-1)}-u\left(A^{(m-2)}+\cdots+A^{\prime}+A\right)=0,
$$

with initial conditions $A(0)=1, A^{\prime}(0)=-1$, and $A^{(i)}(0)=0$ for $2 \leq i \leq m-2$. Equation (2) is now obtained making the substitution $u=u-1$ and using Theorem 1.1 .

\subsection{Chain patterns}

The above proof of Theorem 2.1 can be generalized to any pattern $\sigma$ for which the poset satisfied by the entries of $\pi$ in every cluster is a chain. Recall that a poset is a chain if it is a linear order, i.e., all its elements are comparable.

Definition 2.2 We say that $\sigma \in \mathcal{S}_{m}$ is a chain pattern if all the posets in $\mathcal{P}^{\sigma}$ are chains.

The following generalization can be proved along the lines of the proof of Theorem 2.1 .

Theorem 2.3 Let $m \geq 3$, and let $\sigma \in \mathcal{S}_{m}$ be a chain pattern. Let $\omega(z):=\omega_{\sigma}(u, z)$. Then $\omega$ is the solution of

$$
\omega^{(m-1)}+(1-u) \sum_{d \in O_{\sigma}} \omega^{(m-d-1)}=0
$$

with $\omega(0)=1, \omega^{\prime}(0)=-1, \omega^{(i)}(0)=0$ for $2 \leq i \leq m-2$.

An example of a chain pattern is the pattern $123 \ldots(s-1)(s+1) s(s+2)(s+3) \ldots(2 s)$, for arbitrary $s \geq 3$. In this case, $O_{\sigma}=\{s, s+1, \ldots, 2 s-1\}$. 
Corollary 2.4 Let $s \geq 3$, let $\sigma=123 \ldots(s-1)(s+1) s(s+2)(s+3) \ldots(2 s)$, and let $\omega(z):=\omega_{\sigma}(u, z)$. Then $\omega$ is the solution of

$$
\omega^{(2 s-1)}+(1-u)\left(\omega^{(s-1)}+\cdots+\omega^{\prime}+\omega\right)=0
$$

with $\omega(0)=1, \omega^{\prime}(0)=-1, \omega^{(i)}(0)=0$ for $2 \leq i \leq 2 s-2$.

A more general family of chain patterns can be obtained as follows. Given $\sigma \in \mathcal{S}_{m}$, let $r \geq 0$ be the largest index such that $\sigma_{1} \sigma_{2} \ldots \sigma_{r}=12 \ldots r$, let $s \geq 0$ be the largest such that $\sigma_{m-s+1} \ldots \sigma_{m-1} \sigma_{m}=$ $(m-s+1) \ldots(m-1) m$, let $a \geq 1$ be the largest such that $\sigma_{1} \sigma_{2} \ldots \sigma_{a}$ is increasing, let $b \geq 1$ be the largest such that $\sigma_{m-b+1} \ldots \sigma_{m-1} \sigma_{m}$ is increasing, and let $c=\min \{a, b\}$.

Corollary 2.5 Let $\sigma \in \mathcal{S}_{m} \backslash\{12 \ldots m\}$, and let $r, s, a, b, c$ be defined as above. Suppose that $r, s \geq 1$ and $r+s \geq c+1$, and that $O_{\sigma} \cap\{1,2, \ldots, m-c-1\}=\emptyset$ (i.e., $\sigma$ can only overlap with itself at the initial and final increasing runs). Let $\omega(z):=\omega_{\sigma}(u, z)$. Then $\omega$ is the solution of

$$
\omega^{(m-1)}+(1-u)\left(\omega^{(c-1)}+\cdots+\omega^{\prime}+\omega\right)=0
$$

with $\omega(0)=1, \omega^{\prime}(0)=-1, \omega^{(i)}(0)=0$ for $2 \leq i \leq m-2$.

For example, for $\sigma=123546$, we have $r=3, s=1, a=4, b=2, c=2, O_{\sigma}=\{4,5\}$, and for $\sigma=124536$ all the parameters are the same except that $r=2$. By Corollary 2.5. both $\omega_{123546}(u, z)$ and $\omega_{124536}(u, z)$ satisfy $\omega^{(5)}+(1-u)\left(\omega^{\prime}+\omega\right)=0$, and in particular $P_{123546}(u, z)=P_{124536}(u, z)$. This proves a conjecture of Nakamura [11].

\section{Non-overlapping patterns}

We say that $\sigma \in \mathcal{S}_{m}$ is non-overlapping if $O_{\sigma}=\{m-1\}$, that is, two occurrences of $\sigma$ cannot overlap in more than one position. We assume in this section that $m \geq 2$. In [1], Bóna gives asymptotic estimates on the number of non-overlapping permutations of length $m$, showing in particular that there is a positive fraction of them. In [6, Theorem 3.2], the authors used binary trees to enumerate occurrences of nonoverlapping patterns of the form $\sigma=12 \ldots(b-1) \tau b$, where $2 \leq b<m$, and $\tau$ is any permutation of $\{b+1, b+2, \ldots, m\}$. In fact, the formula holds for slightly more general patterns, as noted in [3], namely non-overlapping patterns with $\sigma_{1}=1$.

Theorem 3.1 (a weaker version appears in [6]) Let $\sigma \in \mathcal{S}_{m}$ be a non-overlapping pattern with $\sigma_{1}=1$, let $b=\sigma_{m}$, and let $\omega(z):=\omega_{\sigma}(u, z)$. Then $\omega$ is the solution of

$$
\omega^{(b)}+(1-u) \frac{z^{m-b}}{(m-b) !} \omega^{\prime}=0
$$

with $\omega(0)=1, \omega^{\prime}(0)=-1, \omega^{(i)}(0)=0$ for $2 \leq i \leq b-1$.

For the special case of $b=2$, that is, when $\sigma=1 \tau 2$, we have the explicit expression

$$
\omega_{\sigma}(u, z)=1-\int_{0}^{z} e^{(u-1) \frac{v^{m-1}}{(m-1) !}} d v,
$$

which can be easily checked to be the solution of equation (5). 
Given an arbitrary non-overlapping pattern, let $a=\sigma_{1}$ and $b=\sigma_{m}$, and note that $a \neq b$. Without loss of generality we can assume that $a<b$, since $\sigma$ and its reversal have the same cluster numbers. A $k$-cluster $\left(\pi ; i_{1}, i_{2}, \ldots, i_{k}\right)$ with respect to $\sigma$ has length $n=k(m-1)+1$ and satisfies $i_{j+1}-i_{j}=m-1$ for all $j$. We have that $\mathcal{I}_{n, k}^{\sigma}=\{(1, m, 2 m-1,3 m-2, \ldots,(k-1)(m-1)+1)\}$, and $\mathcal{P}_{n, k}^{\sigma}$ consists of exactly one poset $P$. To find the shape of $P$, denote by $\varsigma \in \mathcal{S}_{m}$ the inverse of $\sigma$, and observe that the first $m$ entries of $\pi$ must satisfy $\pi_{\varsigma_{1}}<\pi_{\varsigma_{2}}<\cdots<\pi_{\varsigma_{m}}$ to form an occurrence of $\sigma$. Similarly, the entries in positions between $m$ and $2 m-1$ must satisfy $\pi_{\varsigma_{1}+m-1}<\pi_{\varsigma_{2}+m-1}<\cdots<\pi_{\varsigma_{m}+m-1}$. Note that $\pi_{\varsigma_{b}}=\pi_{m}=\pi_{\varsigma_{a}+m-1}$ appears in both lists of inequalities. Repeating this argument for each of the $k$ occurrences of $\sigma$ in the cluster, we see that $P$ is the poset in Figure 1 . Note that this poset depends only on $\sigma_{1}$ and $\sigma_{m}$ but not on the other entries of $\sigma$, and hence so do the cluster numbers. It follows, using Theorem 1.1, that for a non-overlapping pattern $\sigma \in \mathcal{S}_{m}$, the generating function $P_{\sigma}(u, z)$ depends only on $\sigma_{1}$ and $\sigma_{m}$. This fact was recently observed by Dotsenko and Khoroshkin [3] and by Remmel [14].
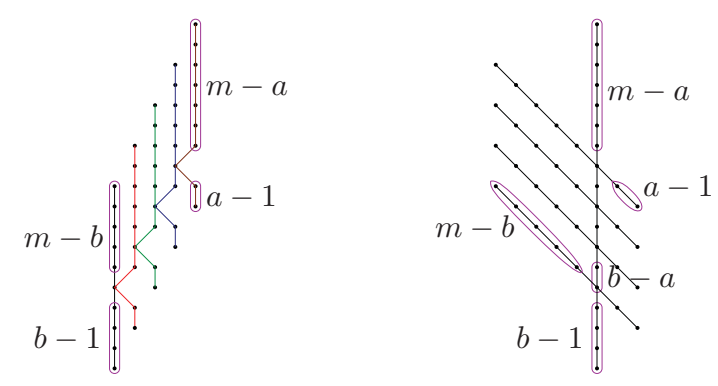

Fig. 1: The poset corresponding to clusters of a non-overlapping pattern $\sigma \in \mathcal{S}_{m}$ with $\sigma_{1}=a$ and $\sigma_{m}=b$.

Proof of Theorem 3.1: The above description of the clusters when $\sigma_{1}=1$ can be used to show that the OGF $\widehat{R}_{\sigma}$ satisfies the differential equation

$$
\widehat{R}_{\sigma}(u, x)=u x^{m}+\frac{u x^{m}}{(m-b) !} \frac{\partial^{m-b}}{\partial x^{m-b}}\left(x^{m-b-1} \widehat{R}_{\sigma}(u, x)\right) .
$$

This gives a differential equation for $\widehat{A}(u, x)=1-x-\widehat{R}_{\sigma}(u, x)$, which then, applying the operator $L$ and using Lemma 1.2 , becomes

$$
A(u, z)=1-z+\frac{u}{(m-b) !} I^{b}\left(z^{m-b} \frac{\partial}{\partial z} A(u, z)\right)
$$

where $A(u, z)=1-z-R_{\sigma}(u, z)$. Differentiating $b$ times, making the change of variable $u=u-1$, and using Theorem 1.1 , we get the differential equation 5 for $\omega_{\sigma}(u, z)$.

Using similar ideas, we have obtained differential equations satisfied by $\omega_{\sigma}(u, z)$ for certain nonoverlapping patterns, such as 12534 or 13254, which are also covered by [8, Corollary 3.9]. Additionally, we can prove two conjectures of Nakamura [11], stating that $P_{123645}(u, z)=P_{124635}(u, z)$ (their inverses satisfy $\omega^{(5)}+(1-u) z\left(\omega^{\prime \prime}+\omega^{\prime}\right)=0$ ) and $P_{132465}(u, z)=P_{142365}(u, z)$ (their inverses satisfy $\left.\omega^{(5)}+(1-u)\left(\omega^{\prime \prime}+z \omega^{\prime}\right)=0\right)$. 


\section{The pattern 1324 and generalizations}

\subsection{The pattern 1324}

This pattern, which is neither non-overlapping nor a chain pattern, cannot be solved with the techniques from [6] or [8], but it has been considered in [3, 9]. In [3], Dotsenko and Khoroshkin give a recurrence for its cluster numbers $r_{n, k}$. In [9], Liese and Remmel use a technique developed in [10] to obtain an ordinary generating function that is equivalent to $\widehat{R}_{1324}(-1, x)$. Here we find the differential equation satisfied by $\omega_{1324}(u, z)$.

Theorem 4.1 Let $\omega(z)=\omega_{1324}(u, z)$. Then $\omega$ is the solution of

$$
\begin{aligned}
& z \omega^{(5)}-(v z-3) \omega^{(4)}-3 v(2 z+1) \omega^{(3)}+v((4 v+1) z-6) \omega^{\prime \prime}+v(8 v z-3) \omega^{\prime}+4 v^{2} z \omega=0 \\
& \text { with } \omega(0)=1, \omega^{\prime}(0)=-1, \omega^{\prime \prime}(0)=\omega^{(3)}(0)=0, \text { and } v=u-1 .
\end{aligned}
$$

Proof: In a cluster $\left(\pi ; i_{1}, \ldots, i_{k}\right)$ with respect to 1324 , we have $i_{j+1}-i_{j} \in O_{1324}=\{2,3\}$ for all $j$. Consider first $k$-clusters of length $n$ where $i_{j+1}-i_{j}=2$ for all $j$, i.e., $\left(i_{1}, \ldots, i_{k}\right)=(1,3,5, \ldots, 2 k+1) \in$ $\mathcal{I}_{n, k}^{\sigma}$, where $n=2 k+2$. The poset in $\mathcal{P}_{n, k}^{\sigma}$ corresponding to this choice of indices is drawn in Figure 2 (when $k=1$, this poset is just a chain). Linear extensions of this poset are in bijection with Dyck paths with $2 k$ steps, so their number is the Catalan number $C_{k}=\frac{1}{k+1}\left(\begin{array}{c}2 k \\ k\end{array}\right)$.

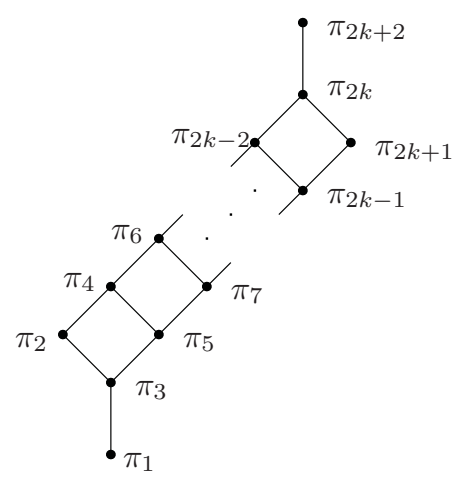

Fig. 2: The order relationships in $k$-clusters with respect to 1324 where neighboring occurrences overlap in two positions.

A cluster $\left(\pi ; i_{1}, \ldots, i_{k}\right)$ may contain two neighboring occurrences of 1324 that overlap in only one entry, i.e., $i_{j+1}-i_{j}=3$ for some $j$. In this case, that entry $\pi_{i_{j+1}}$ is larger than all the entries of $\pi$ to its left and smaller than all the entries to its right. In general, a poset in $\mathcal{P}_{n, k}^{\sigma}$ consists of a tower of pieces isomorphic to the poset in Figure 2, where the top element of each piece is identified with the bottom element of the piece immediately above. A linear extension of the poset is uniquely determined by giving a linear extension for each one of the pieces, since there are no incomparable elements in different pieces. It follows that the OGF for the cluster numbers is

$$
\widehat{R}_{1324}(u, x)=\frac{x}{1-\sum_{k \geq 1} C_{k} u^{k} x^{2 k+1}}-x=\frac{x}{1+x-x C\left(u x^{2}\right)}-x .
$$


where $C(x)=\sum_{k \geq 0} C_{k} x^{k}=\frac{1-\sqrt{1-4 x}}{2 x}$. To be able to apply Theorem 1.1, we need the EGF for the cluster numbers. From equation $\sqrt{6}$ and the fact that $C(x)=1+x C(x)^{2}$, we deduce that $\widehat{A}(u, x)=$ $1-x-\widehat{R}_{1324}(u, x)$ satisfies the algebraic equation

$$
\left(1-u x(1+x)^{2}\right) \widehat{A}(u, x)^{2}+(2 u x(1+x)+x-2) \widehat{A}(u, x)-u x-x+1=0 .
$$

It follows that $\widehat{A}(u, x)$ satisfies a linear differential equation, which we have found using the Maple package gfun. Applying to it the operator $L$ and using Lemma 1.2 , we get

$$
\begin{aligned}
\left(4 u^{2} I^{5}+8 u^{2} I^{4}\right. & \left.+\left(4 u^{2}-u\right) I^{3}-6 t I^{2}-t I+1\right) z \frac{\partial}{\partial z} A(u, z) \\
& +\left(4 u^{2} I^{5}-4 u^{2} I^{3}-2 t I^{3}+6 t I^{2}-1\right) A(u, z)+\left(4 u^{2}+2 u\right) \frac{z^{3}}{6}-7 u \frac{z^{2}}{2}+1=0 .
\end{aligned}
$$

Differentiating (7) four times with respect to $z$, we obtain an equation for $A(z)=A(u, z)$, namely

$$
z A^{(5)}-(u z-3) A^{(4)}-3 u(2 z+1) A^{(3)}+u((4 u-1) z-6) A^{\prime \prime}+u(8 u z-3) A^{\prime}+4 u^{2} z A=0,
$$

with initial conditions $A(0)=1, A^{\prime}(0)=-1, A^{\prime \prime}(0)=0, A^{(3)}(0)=0$. Making the substitution $u=$ $u-1$ and using Theorem 1.1, we obtain an equation for $\omega$.

\subsection{The pattern $134 \ldots(s+1) 2(s+2)(s+3) \ldots(2 s)$}

The method that we used to find a differential equation satisfied by $\omega_{1324}(u, z)$ can be generalized to the pattern $\sigma=134 \ldots(s+1) 2(s+2)(s+3) \ldots(2 s)$ for arbitrary $s \geq 2$.

Theorem 4.2 Fix $s \geq 2$, and let $\sigma=134 \ldots(s+1) 2(s+2)(s+3) \ldots(2 s)$. Then

$$
\widehat{R}_{\sigma}(u, x)=\frac{x^{s}\left(B\left(u x^{s}\right)-1\right)}{1-\left(x+x^{2}+\cdots+x^{s-1}\right)\left(B\left(u x^{s}\right)-1\right)},
$$

where

$$
B(x)=\sum_{k \geq 0} \frac{1}{(s-1) k+1}\left(\begin{array}{c}
s k \\
k
\end{array}\right) x^{k} .
$$

The proof is based on the fact that in any cluster $\left(\pi ; i_{1}, \ldots, i_{k}\right)$ with respect to $\sigma$, we have $i_{j+1}-i_{j} \in$ $O_{\sigma}=\{s, s+1, \ldots, 2 s-1\}$ for all $j$, and the overlaps that create incomparable elements in the poset are between neighboring occurrences of $\sigma$ that share $s$ entries. We call a $k$-cluster of length $n=(k+1) s$ where $i_{j+1}-i_{j}=s$ for all $j$ a dense cluster. The number of dense $k$-clusters is the number of linear extensions of the associated poset, which are in bijection with lattice paths from $(0,0)$ to $(k s, 0)$ with steps $u=(1, s-1)$ and $d=(1,-1)$ that do not go below the $x$-axis. This is why the generating function $B(x)$ appears in the previous statement.

Example 1. Let $\sigma=134256$, which is the case $s=3$. From Theorem 4.2, we obtain using Maple that $\widehat{A}(u, x)=1-x-\widehat{R}_{\sigma}(u, x)$ satisfies a differential equation of the form

$$
p_{0}(u, x) x^{2} \frac{\partial^{2}}{\partial x^{2}} \widehat{A}(u, x)+p_{1}(u, x) x \frac{\partial}{\partial x} \widehat{A}(u, x)+p_{2}(u, x) \widehat{A}(u, x)+\sum_{i=1}^{12} c_{i}(u) x^{i}=0,
$$


where the $p_{i}(u, x)$ and the $c_{i}(u)$ are polynomials, and the $p_{i}(u, x)$ have degree 16 in $x$. Applying the transformation $L$ and using Lemma 1.2 we obtain the following equation for $A(u, z)$ :

$$
p_{0}(u, I) z^{2} \frac{\partial^{2}}{\partial z^{2}} A(u, z)+p_{1}(u, I) z \frac{\partial}{\partial z} A(u, z)+p_{2}(u, I) A(u, z)+\sum_{i=1}^{12} c_{i}(u) \frac{z^{i}}{i !}=0 .
$$

Differentiating 16 times with respect to $z$, we get a linear differential equation for $A(u, z)$, and hence also for $\omega_{\sigma}(u, z)$. It is a differential equation of order 18 with polynomial coefficients.

\section{Other patterns of length four}

\subsection{The pattern 1423}

As in the case of the pattern 1324, we have that $O_{1423}=\{2,3\}$. In this case, for each $k \geq 1$ there is a unique $k$-cluster $\left(\pi ; i_{1}, \ldots, i_{k}\right)$ of length $n=2 k+2$ where $i_{j+1}-i_{j}=2$ for all $j$, because in this case, the poset $P_{n, i_{1}, \ldots, i_{k}}^{1423}$ is a chain $\pi_{1}<\pi_{3}<\pi_{5}<\cdots<\pi_{2 k+1}<\pi_{2 k+2}<\cdots<\pi_{4}<\pi_{2}$.

A general $k$-cluster consists of blocks of marked occurrences that overlap in two positions as above, where the last occurrence in each block overlaps the first occurrence of the next block in one position (so that $i_{j+1}-i_{j}=3$ ). If the first such block has $k_{1}$ occurrences of 1423 , then, in the corresponding poset, the block forms a chain with $2 k_{1}+2$ elements. The element just above the middle of the chain is $\pi_{2 k_{1}+2}$, which is also the first entry of the second block, and thus the bottom element of another chain with $2 k_{2}+2$ elements.

It follows that the cluster numbers satisfy the recurrence

$$
r_{n, k}=\sum_{i=2}^{\lfloor n / 2\rfloor}\left(\begin{array}{c}
n-i-1 \\
i-1
\end{array}\right) r_{n-2 i+1, k-i+1}
$$

with initial conditions $r_{1,0}=1$ and $r_{i, j}=0$ for $i \leq 3$ in all other cases. Multiplying (9) by $(-1)^{k}$ on both sides, summing over all $k$, and letting $b_{n}=\sum_{k}(-1)^{k} r_{n, k}$, we obtain a corresponding recurrence for the $b_{n}$. If we let $B(x)=1+\sum_{n \geq 1} b_{n} x^{n}=1+x+\widehat{R}_{1423}(-1, x)$, it satisfies the functional equation

$$
B(x)=1+\frac{x}{1+x} B\left(\frac{x}{1+x^{2}}\right) .
$$

Although it is straightforward to expand this equation to recover (9), we had to use a generating tree with three labels for the set of clusters to find this equation. Backed by numerical computations by Mireille Bousquet-Mélou using the Maple package gfun, we conjecture that $B(x)$ is not D-finite. This is equivalent to the following statement.

Conjecture 5.1 The generating function $\omega_{1423}(0, z)$ is not D-finite.

Proving this conjecture would give the first known instance of a pattern $\sigma$ for which $\omega_{\sigma}(0, z)=$ $1 / P_{\sigma}(0, z)$ is not D-finite. This becomes particularly interesting when compared with the related conjecture of Gessel, later extended by Noonan and Zeilberger [12], that for any classical pattern $\sigma$ (i.e., where occurrences are not restricted to consecutive positions), the generating function for the number of 
permutations avoiding $\sigma$ is D-finite. Note also that $P_{\sigma}(0, z)$ is already not D-finite for $\sigma=123$, since its denominator has a factor $\cos (3 z / 2+\pi / 6)$ [6], hence $P_{123}(0, z)$ has infinitely many singularities.

A similar argument to the one used for the pattern 1423 shows that $P_{154263}(u, z)=P_{165243}(u, z)$, since the corresponding posets are isomorphic. This proves another conjecture of Nakamura [11], and completes the classification of inequivalent patterns of length up to 6 .

\subsection{The pattern 2143}

Again we have that $O_{2143}=\{2,3\}$. Similarly to the case of the pattern $1423, k$-clusters with respect to 2143 can be broken into blocks in such a way that inside that each block, adjacent occurrences overlap in two positions, and each block overlaps with the next in one position. Let $r_{n, k, \ell}$ be the number of $k$-clusters of length $n$ where $\pi_{1}=\ell+2$. For these refined cluster numbers, we obtain the recurrence

$$
r_{n, k, \ell}=\delta_{n, k, \ell}+\sum_{i=1}^{n / 2} \sum_{j=\ell-1}^{k-2}(n-2 i-j)(\ell+1)\left(\begin{array}{c}
2 i+j-\ell-3 \\
2 i-4
\end{array}\right) r_{n-2 i+1, k-i+1, j}
$$

where $\delta_{n, k, \ell}$ equals 1 if $n=2 k+2$ and $\ell=0$, and 0 otherwise.

\section{Asymptotic results}

The growth constant of a consecutive pattern $\sigma$ is defined as

$$
\rho_{\sigma}=\lim _{n \rightarrow \infty}\left(\frac{\alpha_{n}(\sigma)}{n !}\right)^{1 / n}
$$

where $\alpha_{n}(\sigma)$ is the number of permutations in $\mathcal{S}_{n}$ that avoid $\sigma$. This limit always exists, as shown in [5].

Proposition 6.1 ([5]) For every $\sigma \in \mathcal{S}_{m}, m \geq 3$, there are constants $0<c<d<1$ such that $c^{n} n$ ! $<$ $\alpha_{n}(\sigma)<d^{n} n !$ for all $n$. Additionally, $\lim _{n \rightarrow \infty}\left(\alpha_{n}(\sigma) / n !\right)^{1 / n}$ exists, and it is between 0.7839769 and 1 .

The growth constant was determined in [6] for all patterns of length three and several patterns of length four. The conjecture that the monotone pattern is always dominating [6] is still open.

Conjecture 6.2 ([6]) For any $\sigma \in \mathcal{S}_{m}$, we have $\rho_{\sigma} \leq \rho_{12 \ldots m}$.

We can prove that the above conjecture is true for non-overlapping patterns, which as mentioned before represent a positive fraction of all patterns.

Theorem 6.3 Let $\sigma \in \mathcal{S}_{m}(m \geq 3)$ be a non-overlapping pattern. There is an $n_{0}$ such that for all $n \geq n_{0}$

$$
\alpha_{n}(\sigma)<\alpha_{n}(12 \ldots m)
$$

An important recent result of Ehrenborg, Kitaev and Perry [4], proved using methods from spectral theory is the following:

Theorem 6.4 ([4]) For every pattern $\sigma, \alpha_{n}(\sigma) / n !=\gamma \rho_{\sigma}^{n}+O\left(r^{n}\right)$, where $\gamma, \rho_{\sigma}$ and $r$ are positive constants such that $\rho_{\sigma}>r$. 
In all cases we have been able to solve, $\omega_{\sigma}(0, z)$ is an entire function and so the EGF $P_{\sigma}(0, z)$ is meromorphic. Theorem 6.4 implies that $\rho_{\sigma}$ must then be a simple zero of $\omega_{\sigma}(0, z)$, hence a simple pole of $P_{\sigma}(0, z)$. Concerning the nature of the function $\omega_{\sigma}(u, z)$, we have proved that it is entire in several cases.

Theorem 6.5 For any non-overlapping pattern $\sigma \in \mathcal{S}_{m}$ and for any fixed $u \in \mathbb{C}, \omega_{\sigma}(u, z)$ is an entire function of $z$.

Theorem 6.6 If $\sigma \in \mathcal{S}_{m}$ satisfies $\sigma_{1}=1$, then for any fixed $u \in \mathbb{C}, \omega_{\sigma}(u, z)$ is an entire function of $z$.

On the other hand, $\omega_{\sigma}(u, z)$ is not always entire for all values of $u$.

Proposition 6.7 The function $\omega_{2413}(2, z)$ is not entire.

\section{References}

[1] M. Bóna, Non-overlapping permutation patterns, Pure Mathematics and Its Applications, to appear.

[2] D.C. Brydges, T. Spencer, Self-avoiding walks in 5 or more dimensions, Comm. Math. Phys. 97 (1985), 125-148.

[3] V. Dotsenko and A. Khoroshkin, Anick-type resolutions and consecutive pattern avoidance, preprint, arXiv: 1002.2761 .

[4] R. Ehrenborg, S. Kitaev and P. Perry, A spectral approach to consecutive pattern avoiding permutations, preprint, arXiv:1009.2119.

[5] S. Elizalde, Asymptotic enumeration of permutations avoiding generalized patterns, Adv. in Appl. Math. 36 (2006), 138-155.

[6] S. Elizalde and M. Noy, Consecutive patterns in permutations, Adv. Appl. Math. 30 (2003), 110-123.

[7] I.P. Goulden, D.M. Jackson, Combinatorial Enumeration, John Wiley \& Sons, Inc., New York, 1983.

[8] A. Khoroshkin and B. Shapiro, Using homological duality in consecutive pattern avoidance, Electron. J. Combin. 18 (2011), \#P9.

[9] J. Liese and J. Remmel, Generating functions for permutations avoiding a consecutive pattern, Ann. Comb. 14 (2010), 123-141.

[10] A. Mendes and J. Remmel, Permutations and words counted by consecutive patterns, Adv. Appl. Math. 37 (2006), 443-480.

[11] B. Nakamura, Computational Approaches to Consecutive Pattern Avoidance in Permutations, Pure Mathematics and Applications, to appear, arXiv:1102.2480.

[12] J. Noonan and D. Zeilberger, The enumeration of permutations with a prescribed number of "forbidden" patterns, Adv. in Appl. Math. 17 (1996), 381-407.

[13] J. Noonan and D. Zeilberger, The Goulden-Jackson cluster method: extensions, applications and implementations, J. Differ. Equations Appl. 5 (1999), 355-377.

[14] J. Remmel, personal communication, August 2010. 